\title{
Thick and thin: Bridging the gap to a better understanding of apical thinning
}

\author{
Howard M. Julien, MD, MPH, ${ }^{\mathrm{a}}$ and Paco E. Bravo, $\mathrm{MD}^{\mathrm{a}, \mathrm{b}}$ \\ a Division of Cardiology, Department of Medicine, Perelman School of Medicine, University of \\ Pennsylvania, Philadelphia, PA \\ b Divisions of Nuclear Medicine and Cardiology, Departments of Radiology and Medicine, \\ Perelman School of Medicine, University of Pennsylvania, Philadelphia, PA
}

Received Sep 14, 2018; accepted Sep 17, 2018

doi: $10.1007 / \mathrm{s} 12350-018-1451-0$

\section{See related article, pp. 452-460}

The presence of reduced radiotracer uptake in the left ventricular (LV) apex on myocardial perfusion imaging (MPI) - commonly known as "apical thinning" - introduces diagnostic uncertainty for clinicians who aim to discern pathologic decreases in radiotracer uptake from anatomic variants and/or artifacts. Although apical thinning most commonly results in a fixed "defect" in the setting of normal apical wall motion, interpreters are often faced with a diagnostic dilemma when its presence is severe, ${ }^{1}$ extends to the anteroapical wall and/or is completely/partially reversible when comparing stress images to those obtained at rest. $^{2}$ Apical thinning also appears to be a very common finding. ${ }^{3}$ In a study of 102 subjects (46 with angiographic coronary artery disease and 56 normal subjects) undergoing single-photon emission computed tomography (SPECT) apical thinning of any degree (as defined on visual scale) was found in $63 \%$ of normal subjects on attenuation-corrected images (graded severe in 5\%), although the prevalence appeared to be somewhat higher (71\%) on the non-corrected SPECT images. ${ }^{1}$ The clinical significance of these findings is especially relevant as interpreters of nuclear imaging studies across modalities attempt to elucidate the etiology of this well-

Reprint requests: Paco E. Bravo, MD, Divisions of Nuclear Medicine and Cardiology, Departments of Radiology and Medicine, Perelman School of Medicine, University of Pennsylvania, 3400 Civic Center Boulevard, 11-154 South Pavilion, Philadelphia, PA 19104; paco.bravo@uphs.upenn.edu

J Nucl Cardiol 2020;27:461-4.

1071-3581/\$34.00

Copyright (C) 2018 American Society of Nuclear Cardiology. described, but incompletely understood, phenomenon. Proposed mechanisms include a base-to-apex gradient in (both relative and absolute) myocardial blood flow $(\mathrm{MBF}),{ }^{4}$ variations in image acquisition and processing, in addition to partial volume averaging resulting from true anatomic thinning. For instance, one notable image processing technique that has been implicated in the presence of apical thinning is the application of computed tomography (CT) attenuation correction processing. Phantom studies have demonstrated that low-dose CT attenuation correction exaggerates the finding of apical thinning, ${ }^{5}$ whereas others have not found attenuation correction to affect or worsen apical thinning (Figure 1). ${ }^{1}$ Similarly, time-of-flight ordered subset expectation maximization image reconstruction has been noted to result in emphasis of apical thinning on positron emission tomography (PET) perfusion scans with ${ }^{13} \mathrm{~N}$-Ammonia. ${ }^{6}$

On the other hand, for decades, post-mortem studies have established the remarkable geometry of the LV apex. ${ }^{7}$ In a gross and histologic series of 60 hearts free of local disease at necropsy, Bradfield et al. found that the mean wall thickness of the LV apex at its thinnest point was $1.3 \pm 0.7 \mathrm{~mm}$. In fact, the apical thin point measured $\leq 2 \mathrm{~mm}$ in $97 \%$ and $\leq 1 \mathrm{~mm}$ in $67 \%$ of the hearts in this series. This anatomic finding has been confirmed in the modern non-invasive imaging era with multidetector $\mathrm{CT} .{ }^{8,9}$ Ferencik et al. found that the mean thickness of the LV apex at its thinnest point was $2.3 \pm 1.2 \mathrm{~mm}$ from the axial plane or $1.7 \pm 0.7 \mathrm{~mm}$ if measured from the long-axis plane. A thickness of $\leq 3$ $\mathrm{mm}$ was observed in $77 \%$ and $100 \%$ of the hearts from the axial and long-axis plane, respectively. These are measurements that, independent of the study, are below the spatial resolution of the reconstructed axial image of PET $(3.9-5.8 \mathrm{~mm})$ and SPECT $(\sim 10 \mathrm{~mm}$ at $10 \mathrm{~cm})$ systems, and thus would be subject to varying degrees of 


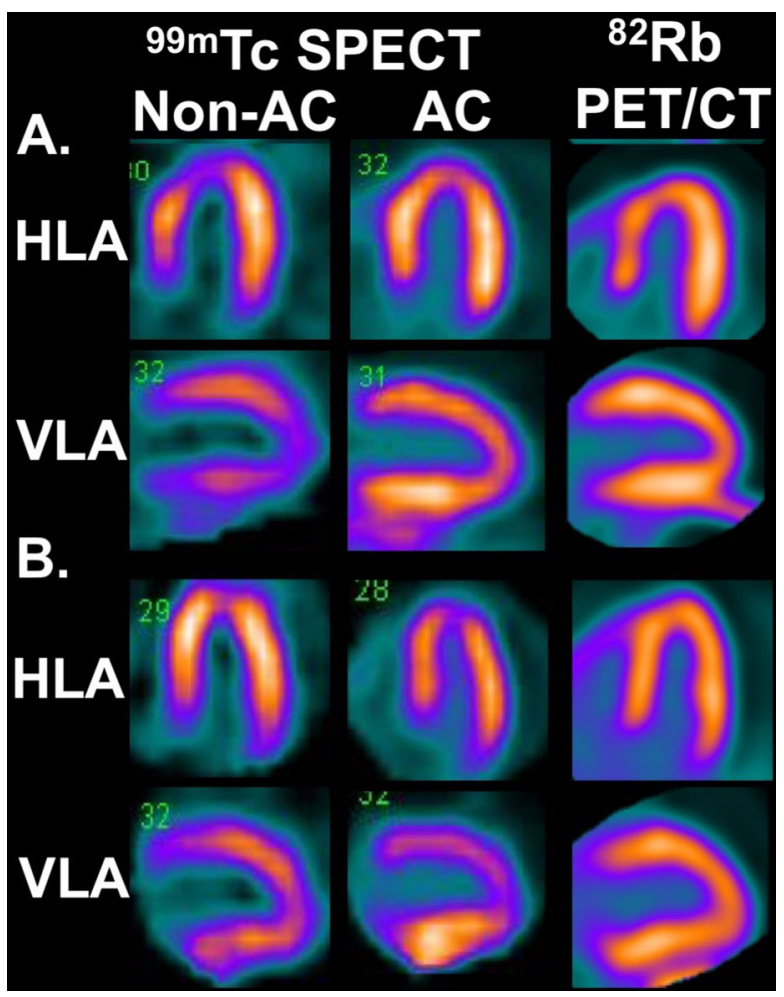

Figure 1. Effect of different radionuclide techniques in the reconstruction of radiotracer distribution within the left ventricular apex. Two patients (A and $\mathbf{B})$ underwent myocardial perfusion with ${ }^{99 \mathrm{~m}} \mathrm{Tc}$-sestamibi SPECT/CT and ${ }^{82} \mathrm{Rb}$ PET/ $\mathrm{CT}$ less than one month apart. In case A, apical thinning is clearly seen with non-attenuation correction (non-AC) SPECT images, subtle with AC SPECT/CT, and absent with PET/CT. In contrast in patient $\mathrm{B}$, apical thinning was more obvious with $\mathrm{AC}$ than with non-AC SPECT, and again was absent with PET/ CT.

partial volume averaging, an inherent limitation to crosssectional imaging in general but particularly relevant to MPI. ${ }^{10}$

To date, this effect had not been studied in the same patient population across imaging modalities. In the present issue, ${ }^{11}$ Steffen et al report the important results of a single-center retrospective analysis of 57 patients without coronary artery disease who underwent MPI with ${ }^{13} \mathrm{~N}$-Ammonia PET as well as prospectively gated contrast-enhanced coronary CT angiography (CTA). The authors compared the base-to-apex changes in myocardial wall thickness derived from CTA with a number of PET markers of myocardial perfusion, including normalized percent tracer uptake (summed, end-diastolic, end-systolic), MBF, and k2 washout rate. They noticed that $93 \%$ of patients had evidence of reduced relative apical tracer uptake (on static/non-gated images) and were considered, thus, as having apical thinning. Interestingly, while they also found a clear base-to-apex gradient in wall thickness and in most PET parameters (except for $\mathrm{k} 2$ washout rate), they failed to observe a significant correlation between apical myocardial thickness and perfusion at the apical segments, hence, suggesting that partial volume average may not the be sole cause of apical thinning on MPI. The study is important from a mechanistic perspective, as it provides new insights into this common imaging phenomenon. However, one must be careful with the interpretation of these results. The fact that no correlation was observed between wall thickness and perfusion (limited to the apical segments) is not a sufficient proof to disregard partial volume as the driving force explaining apical thinning, especially since the range of variation of the analyses within the boundaries of the apical segments may not have been sufficient to identify a correlation coefficient. On the contrary, the study offers enough evidence to further support the critical role of partial volume in contributing to the development of apical thinning as suggested by the following findings: (1) there was a significant gradient in wall thickness from base $(9.0 \mathrm{~cm} \pm 1.6)$, apex $(4.6 \mathrm{~cm} \pm$ $0.7)$, and to the thinnest point of the apex $(2.3 \mathrm{~cm} \pm$ 0.8 ); (2) this gradient was also evidenced in all of the PET parameters known to be affected by partial volume effect including normalized percent tracer uptake and retention-based quantitative MBF; (3) apical tracer uptake improved significantly at end-systole (when wall thickening is the greatest); and (4) myocardial k2 washout, a quantitative PET parameter that is less likely to be affected by partial volume effect, ${ }^{12}$ failed to show a base-to-apex gradient.

Accordingly, the existing evidence suggests that apical thinning in normal individuals is clearly related to a significant drop/change in wall thickness from the mid-apical wall segment transition $(\sim 5 \mathrm{~mm})$ down to thinnest point of the LV apex $(<3 \mathrm{~mm})$, and thus most likely explained by partial volume averaging. Moreover, in our experience, this phenomenon of "wall thinning" is not exclusive to the LV apex and can actually be observed in any wall in which there are significant reductions in regional LV wall thickness (obviously after excluding scarring) relative to normal or hypertrophied LV segments (Figure 2).

Given the potential diagnostic challenges imposed on nuclear cardiologists by the high prevalence of apical thinning during myocardial perfusion imaging, future studies should focus on the investigation and development of more accurate methods for partial volume correction on MPI SPECT and PET systems. This will 


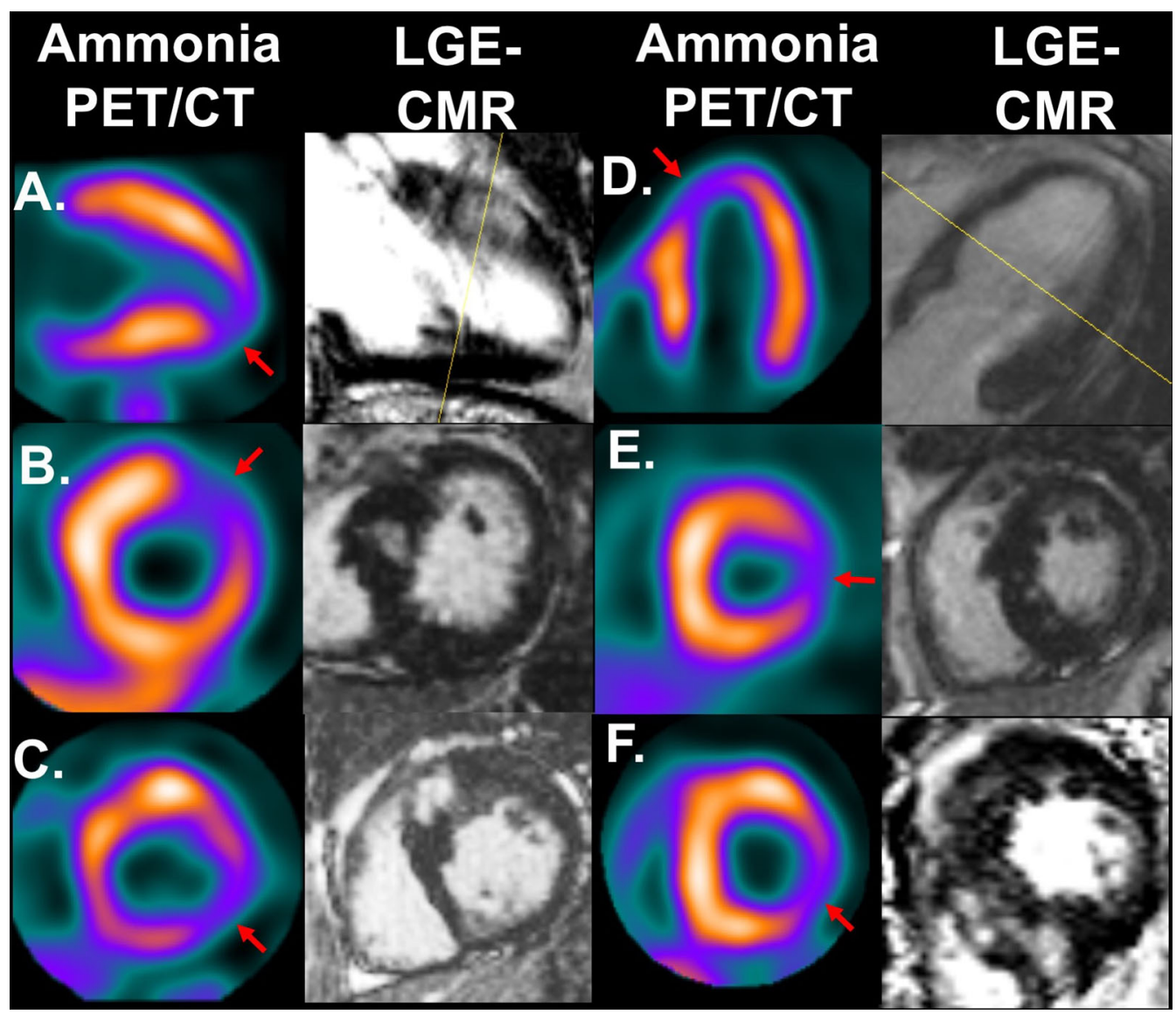

Figure 2. Impact of regional changes in left ventricular (LV) wall thickness on the distribution of radiotracer activity in patients with hypertrophic cardiomyopathy on resting ${ }^{13} \mathrm{~N}$-ammonia PET/CT. Compared to the most hypertrophied LV segments, there is relative reduction in radiotracer (ammonia) activity at the thinnest point (arrows) of the LV, including apical inferior (A), basal anterolateral (B), basal inferolateral (C, F), apical septum (D), and basal lateral wall (E). Please notice that these thin points/regions show no evidence of late gadolinium enhancement (LGE) on corresponding cardiac magnetic resonance (CMR) images, thus, the reduced radiotracer uptake on resting ammonia PET at these sites is not due to scar, but most likely partial volume effect.

translate into better image interpretability and thus improved diagnostic performance.

\section{Disclosure}

The authors have no conflict of interest to disclose.

\section{References}

1. Links JM, Becker LC, Anstett F. Clinical significance of apical thinning after attenuation correction. J Nucl Cardiol 2004;11:2631 .

2. Apostolopoulos DJ, Savvopoulos C. What is the benefit of ctbased attenuation correction in myocardial perfusion spet? Hell $\mathrm{J}$ Nucl Med 2016;19:89-92.
3. Dvorak RA, Brown RK, Corbett JR. Interpretation of spect/ct myocardial perfusion images: Common artifacts and quality control techniques. Radiographics 2011;31:2041-57.

4. Hernandez-Pampaloni M, Keng FY, Kudo T, Sayre JS, Schelbert HR. Abnormal longitudinal, base-to-apex myocardial perfusion gradient by quantitative blood flow measurements in patients with coronary risk factors. Circulation 2001;104:527-32.

5. Purser NJ, Armstrong IS, Williams HA, Tonge CM, Lawson RS. Apical thinning: Real or artefact? Nucl Med Commun 2008;29:382-9.

6. Tomiyama T, Ishihara K, Suda M, Kanaya K, Sakurai M, Takahashi $\mathrm{N}$, et al. Impact of time-of-flight on qualitative and quantitative analyses of myocardial perfusion pet studies using (13)n-ammonia. J Nucl Cardiol. 2015;22:998-1007.

7. Bradfield JW, Beck G, Vecht RJ. Left ventricular apical thin point. Br Heart J 1977;39:806-9.

8. Ferencik M, Abbara S, Hoffmann U, Cury RC, Brady TJ, Achenbach S. Left ventricular thin-point detection using 
multidetector spiral computed tomography. Am J Cardiol 2004;93:949-51.

9. Johnson KM, Johnson HE, Dowe DA. Left ventricular apical thinning as normal anatomy. J Comput Assist Tomogr 2009;33:334-7.

10. Okuda K, Nakajima K, Matsuo S, Wakabayashi H, Taki J, Kinuya S. Cause of apical thinning on attenuation-corrected myocardial perfusion spect. Nucl Med Commun 2011;32:1033-9.

11. Steffen DA, Giannopoulos AA, Grossmann M, Messerli M, Schwyzer M, Grani C, et al. Apical thinning: Relations between myocardial wall thickness and apical left ventricular tracer uptake as assessed with positron emission tomography myocardial perfusion imaging. J Nucl Cardiol 2018. https://doi.org/10.1007/ s12350-018-1397-2.

12. Lubberink M, Harms HJ, Halbmeijer R, de Haan S, Knaapen P, Lammertsma AA. Low-dose quantitative myocardial blood flow imaging using 150 -water and pet without attenuation correction. $\mathrm{J}$ Nuclear Med 2010;51:575-80. 